\title{
DISCRETE MEASURES AND THE RIEMANN HYPOTHESIS
}

\author{
By Alberto Verjovsky
}

\section{Introduction}

The purpose of this paper is to show that the Riemann hypothesis is equivalent to a problem of the rate of convergence of certain discrete measures defined on the positive real numbers to the measure $\frac{6}{\pi^{2}} u d u$, where $d u$ is Lebesgue measure.

As a motivation consider the following: For each positive real number $y$, let $\mu_{y}$ be the infinite measure on the real line defined by

$$
\mu_{y}=\sum_{n \in \mathbb{Z}} y \delta_{n y}
$$

where $\mathbb{Z}$ denotes the integers and $\delta_{x}$ denotes the Dirac mass at the point $x \in \mathbb{R}$. It follows by the Poisson summation formula that if $f \in C_{c}^{\infty}(\mathbb{R})\left(C_{c}^{\infty}(\mathbb{R})=\right.$ functions $f: \mathbb{R} \rightarrow \mathbb{R}$, of class $C^{\infty}$ and with compact support), then for every $\beta>0$ :

$$
\mu_{y}(f)=\int_{\mathbb{R}} f(t) d t+o\left(y^{\beta}\right), \quad \text { as } y \rightarrow 0 .
$$

This is so because by the Poisson summation formula [B],

$$
y \sum_{n \in \mathbb{Z}} f(n y)=\sum_{n \in \mathbb{Z}} \hat{f}\left(n y^{-1}\right)
$$

where $\hat{f}$ is the Fourier transform of $f$ and, since $f$ is smooth with compact support we have that $\hat{f}$ is of rapid decay at infinity. Hence

$$
y \sum_{n \in \mathbb{Z}} f(n y)=\widehat{f}(0)+o\left(y^{\beta}\right) \quad \text { as } y \rightarrow 0 \text { for all } \beta>0 .
$$

So, as $y \rightarrow 0$, the atoms of $\mu_{y}$ cluster uniformly and $\mu_{y}(f)$ gives a very good approximation of integrals of smooth functions with compact support.

Now let $\mathbb{R}^{\bullet}$ denote the multiplicative group of positive real numbers. For each $y \in \mathbb{R}^{\bullet}$, let us consider the infinite measure, $m_{y}$, defined on smooth functions with compact support in $\mathbb{R}^{\bullet}$, by the formula:

$$
m_{y}(f)=\sum_{n \in \mathbb{N}} y \varphi(n) f\left(y^{\frac{1}{2}} n\right)
$$

where $\mathbb{N}=\{1,2, \ldots\}$ is the set of natural numbers and $\varphi(n)=n \prod_{p \mid n}\left(1-\frac{1}{p}\right)$ is Euler's totient function, which counts the number of integers which are relatively prime to a given integer, and are lesser or equal to that integer. In fact, for every $r \geqq 0, r$ an

Received June 17, 1993 
integer or $r=\infty$, we can consider $m_{y}$ as an element in the dual space of $C_{c}^{r}\left(\mathbb{R}^{\bullet}\right)=$ complex-valued functions $f: \mathbb{R}^{\bullet} \rightarrow \mathbb{C}$, of class $C^{r}$, with compact support.

We will prove the following theorems:

THEOREM A. For every $f \in C_{c}^{0}\left(\mathbb{R}^{\bullet}\right)$ :

$$
\begin{aligned}
m_{y}(f) & =\frac{1}{\zeta(2)} \int_{0}^{\infty} u f(u) d u+\mathcal{O}\left(y^{1 / 2} \log y\right) \\
& =\frac{6}{\pi^{2}} \int_{0}^{\infty} u f(u) d u+\mathcal{O}\left(y^{\frac{1}{2}} \log y\right) \quad \text { as } y \rightarrow 0
\end{aligned}
$$

DeFinition. Let $m_{0}(f)=\int_{0}^{\infty} \frac{6}{\pi^{2}} u f(u) d u$.

THEOREM B. 1) The Riemann hypothesis is true if and only if for every function $f \in C_{c}^{r}\left(\mathbb{R}^{\bullet}\right)$, with $2 \leqq r \leqq \infty$

as $y \rightarrow 0$, for all $\epsilon>0$.

$$
m_{y}(f)=m_{0}(f)+o\left(y^{\frac{3}{4}-\epsilon}\right)
$$

2) Furthermore, if $\alpha \in\left(\frac{1}{2}, \frac{3}{4}\right)$ is such that, for all functions $f \in C_{c}^{2}\left(\mathbb{R}^{\bullet}\right)$ one has,

$$
m_{y}(f)=m_{0}(f)+o\left(y^{\alpha-\epsilon}\right)
$$

as $y \rightarrow 0$, for all $\epsilon>0$, then the Riemann zeta-function has no zeroes in the halfplane $\Re(s)>2(1-\alpha)$. Conversely, if the Riemann zeta-function has no zeroes in the half-plane $\Re(s)>2(1-\alpha)$ then (3) holds for all functions $f \in C_{c}^{2}\left(\mathbb{R}^{\bullet}\right)$.

3) If $f$ is the characteristic function of an interval then:

$$
\varlimsup_{y \rightarrow 0} y^{-\alpha}\left|m_{y}(f)-m_{0}(f)\right|=\infty, \quad \text { if } \alpha>\frac{1}{2} .
$$

Hence $\frac{1}{2}$ is the best possible exponent of the error for some nonsmooth functions.

4) Let the function $F$, with domain in the positive reals be defined by

Then:

$$
F(x)= \begin{cases}1-x & \text { for } x \leqq 1 \\ 0 & \text { for } x>1\end{cases}
$$

$$
m_{y}(F)=\frac{1}{\pi^{2}}+o\left(y^{\frac{1}{2}}\right) \quad \text { as } y \rightarrow 0
$$

and,

$$
\varlimsup_{y \rightarrow 0} y^{-\alpha}\left|m_{y}(F)-m_{0}(F)\right|=\infty, \quad \text { for all } \alpha>\frac{1}{2}
$$

if and only if the Riemann hypothesis is false in the strongest possible sense: there exıst zeroes of Riemann's $\zeta$-function arbitrarily close to the critıcal line $\Re(s)=1$.

If $f=\chi_{[a, b]}$ is the characteristic function of the interval $[a, b], 0<a<b$, then we can also define $m_{y}(f)$ in the obvious manner:

$$
m_{y}(f)=\sum_{a y^{-\frac{1}{2}} \leqq n \leqq b y-\frac{1}{2}} y \varphi(n) .
$$


The measures $m_{y}$ and their connection to the Riemann hypothesis were discovered by the author as a consequence of studying geometrically the beautiful paper [Z] by Don Zagier. The author wrote [V] inspired by this paper which contains a remarkable connection obtained by Zagier between the Riemann Hypothesis and horocyclic measures on the modular orbifold (see also P. Sarnak [S] and E. Ghys [G]). The present paper can be thought of as a continuation of [V].

In order to be as self-contained as possible we will recall some classical and fundamental results.

\section{Preliminaries}

First, let us start by proving formula (2) for characteristic functions. Let $f=\chi_{[a, b]}$ be the characteristic function of the interval $[a, b]$, where $0<a<b$. Then: -

$$
\begin{aligned}
m_{y}(f) & =\sum_{a y^{-\frac{1}{2}} \leqq n \leqq b y-\frac{1}{2}} y \varphi(n)=\frac{1}{2 \zeta(2)}\left[b^{2}-a^{2}\right]+\mathcal{O}\left(y^{\frac{1}{2}} \log y\right) \\
& =\frac{1}{\zeta(2)} \int_{0}^{\infty} u f(u) d u+\mathcal{O}\left(y^{\frac{1}{2}} \log y\right) .
\end{aligned}
$$

The second equality follows from the well-known formula:

$$
\sum_{n \leqq x} \varphi(n)=\frac{3}{\pi^{2}} x^{2}+\mathcal{O}(x \log x), \quad x>1 .
$$

This formula, due to Mertens (1874), can be found, for instance, in Hardy and Wright [HW] or Apostol [A], p. 70.

Thus,

$$
m_{y}(f)=m_{0}(f)+\mathcal{O}\left(y^{\frac{1}{2}} \log y\right) \quad \text { as } y \rightarrow 0 .
$$

If $m_{y}(f)-m_{0}(f)=K_{f}(y)$, then $K_{f}(y)=h_{f}(y) y^{\frac{1}{2}} \log y$ and $h_{f}(y)$ remains bounded as $y \rightarrow 0$ and the bound depends only on the interval $[a, b]$. If $f \in C_{c}^{1}\left(\mathbb{R}^{\bullet}\right)$ then we can apply Abel's summation formula and (6) to obtain:

$$
\begin{aligned}
m_{y}(f) & =\sum_{n \in \mathbb{N}} y \varphi(n) f\left(y^{\frac{1}{2}} n\right)=-\frac{1}{2 \zeta(2)} \int_{0}^{\infty} u^{2} f^{\prime}(u) d u+\mathcal{O}\left(y^{\frac{1}{2}} \log y\right) \\
& =\frac{1}{\zeta(2)} \int_{0}^{\infty} u f(u) d u+\mathcal{O}\left(y^{\frac{1}{2}} \log y\right), \quad \text { as } y \rightarrow 0 .
\end{aligned}
$$

Since any continuous function with compact support in $\mathbb{R}^{\bullet}$ can be uniformly approximated by $C^{1}$ functions with compact support in $\mathbb{R}^{\bullet}$, and the error terms depend only on the support of the functions, we immediately obtain Theorem A. However, Theorem A will also be a consequence of what follows. It is interesting to note that the volume of $P S L(2, \mathbb{R}) / P S L(2, \mathbb{Z})$, with respect to Haar measure, is $\pi^{2} / 3$. As it turns out, Mertens Theorem corresponds to the statement that the ergodic measures of the horocyclic flow which are supported on the periodic orbits and uniformly distributed with respect to arc-length, converge vaguely to Haar measure as the period tends to infinity (see [V] and $[\mathrm{Z}]$ ). 


\subsection{Mellin transform}

To see how naturally the Riemann $\zeta$ function arises in connection with the measures $m_{y}$, let us first recall a classical formula:

$$
\frac{\zeta(s-1)}{\zeta(s)}=\sum_{n \geqq 1} \frac{\varphi(n)}{n^{s}}, \text { for } \Re(s)>2
$$

(see, for instance, [A], p. 229). Let $r \geqq 0$ be an integer or infinity. For each $f \in C_{c}^{r}\left(\mathbb{R}^{\bullet}\right)$ consider the Mellin transform of $m_{y}(f)$ :

$$
\mathcal{M}_{f}(s)=\int_{0}^{\infty} m_{y}(f) y^{s-2} d y
$$

Proposition 2.1.1. The integral defining $\mathcal{M}_{f}(s)$ converges absolutely in the halfplane $\Re(s)>1$ and uniformly in $\Re(s)>1+\epsilon$ for all $\epsilon>0$. Therefore, it defines a holomorphic function on the half plane $\Re(s)>1$.

Proof. Let us suppose that support $(f) \subset[a, b] ; 0<a<b$. Let $\|f\|_{\infty}=\sup _{y \in \mathbb{R}^{\bullet}}$ $|f(y)|$. Then if $\Re(s)>1$, we have, since $\left|m_{y}(f)\right| \leqq A\|f\|_{\infty}$, for some $A>0$ :

$$
\left|\mathcal{M}_{f}(s)\right| \leqq\|f\|_{\infty} A\left(\frac{b^{\sigma-1}}{\sigma-1}\right), \quad \text { where } \sigma=\Re(s) .
$$

Therefore, we have absolute convergence in $\Re(s)>1$, and evidently the convergence is uniform in $\Re(s)>1+\epsilon$ for $\epsilon>0$.

Remarks 2.1.2.

(a) Strictly speaking, equation (8) defines, in classical notation, the Mellin transform of $y^{-1} m_{y}(f)$; however, we will still call it the Mellin transform of $m_{y}(f)$. Let $\left[C_{c}^{r}\left(\mathbb{R}^{\bullet}\right)\right]^{*}$ denote the topological dual of $C_{c}^{r}\left(\mathbb{R}^{\bullet}\right)$. Then the function

$$
\mathcal{M}:,\{\Re(s)>1\} \rightarrow\left[C_{c}^{r}\left(\mathbb{R}^{\bullet}\right)\right]^{*},
$$

given by

$$
s \mapsto \int_{0}^{\infty} m_{y}(\cdot) y^{s-2} d y, \quad \Re(s)>1
$$

defines a weakly holomorphic function. For every $s$ such that $\Re(s)>1, \mathcal{M}$ defines an infinite measure on $\mathbb{R}^{\circ}$. When $r=\infty, \mathcal{M}$ defines a holomorphic function whose values are distributions of finite order. Compare [S]. We will be able to continue $\mathcal{M}$ analytically to obtain a weakly meromorphic function with values in the distribution space of $\mathbb{R}^{\bullet}$.

(b) We notice that for every $0 \leqq r \leqq \infty$, and $f \in C_{c}^{r}\left(\mathbb{R}^{\bullet}\right)$ we have $m_{y}(f)=0$ if $y$ is sufficiently large:

$$
m_{y}(f)=0 \text { for all } y>b, \quad \text { where support }(f) \subset[a, b] .
$$

(c) Via the logarithm, or the exponential, we can transport measures defined on $\mathbb{R}$ to measures defined on $\mathbb{R}^{\bullet}$, and vice versa. Let $m_{y}^{+}=\exp ^{*}\left(m_{y}\right)$ be the measure on the real line obtained by pulling back $m_{y}$ by $\exp : \mathbb{R} \rightarrow \mathbb{R}^{\bullet}$. Then $m_{y}^{+}$is supported on a discrete set of points which is irregularly distributed on the real line and the Dirac masses that define $m_{y}^{+}$are weighted by Euler's function. This 
accounts for the difference between the measures $\mu_{y}$ defined at the beginning of the introduction and $m_{y}$, as far as error terms are concerned. This also establishes a connection between the measures $\left\{m_{y}\right\}_{y>0}$ and Farey sequences as in the wellknown results of Franel ([F]) and Landau ([La]). See ([V]).

Now, let us combine equations (1) and (8) to obtain:

$$
\mathcal{M}_{f}(s)=\int_{0}^{\infty}\left(\sum_{n \in \mathbb{N}} y \varphi(n) f\left(y^{\frac{1}{2}} n\right)\right) y^{s-2} d y ; \quad \Re(s)>1 .
$$

Fix $n \in \mathbb{N}$ and define $\psi_{n}: \mathbb{R}^{\bullet} \rightarrow \mathbb{C}$ by the formula

$$
\psi_{n}(y)=y \varphi(n) f\left(y^{\frac{1}{2}} n\right) \text {. }
$$

Then:

$$
\int_{0}^{\infty} \psi_{n}(y) y^{s-2} d y=\varphi(n) \int_{0}^{\infty} f\left(y^{\frac{1}{2}} n\right) y^{s-1} d y .
$$

Changing variable: $u=y^{\frac{1}{2}} n$, we get:

$$
\int_{0}^{\infty} \psi_{n}(y) y^{s-2} d y=2 \frac{\varphi(n)}{n^{2 s}} \int_{0}^{\infty} f(u) u^{2 s-1} d u ; \quad \Re(s)>1 .
$$

Now, if $\sigma=\Re(s)>2$, we have $\left|\frac{\varphi(n)}{n^{2 s}}\right| \leqq \frac{1}{n^{3}}$. Hence, by the Lebesgue dominated convergence theorem and formula (7) we obtain:

Proposition 2.1.3.

$$
\mathcal{M}_{f}(s)=2 \frac{\zeta(2 s-1)}{\zeta(2 s)} \int_{0}^{\infty} f(u) u^{2 s-1} d u ; \quad \Re(s)>2 .
$$

Furthermore if $F$ is the function defined in Theorem $B$ part 3), then all the above applies so:

$$
\mathcal{M}_{f}(s)=\frac{\zeta(2 s-1)}{s(2 s+1) \zeta(2 s)}
$$

Let

$$
\varphi_{f}(s)=\int_{0}^{\infty} f(u) u^{2 s-1} d u
$$

Since $f$ has compact support it follows that $\varphi_{f}(s)$ is an entire function with derivative

$$
\frac{d}{d s}\left(\varphi_{f}(s)\right)=2 \int_{0}^{\infty} f(u)(\log u) u^{2 s-1} d u
$$

Therefore, we see from (13) that $\mathcal{M}_{f}(s)=\frac{2 \zeta(2 s-1)}{\zeta(2 s)} \varphi_{f}(s)$, can be continued as a meromorphic function to all of $\mathbb{C}$ and we obtain, using the properties of $\zeta$, the following:

Proposition 2.1.4. a) $\mathcal{M}_{f}(s)$ is a meromorphic function with a simple pole at $s=1$ with residue:

$$
\operatorname{Res}_{s=1}\left(\mathcal{M}_{f}(s)\right)=\frac{1}{\zeta(2)} \int_{0}^{\infty} u f(u) d u .
$$

b) All other possible poles of $\mathcal{M}_{f}(s)$ are the negative integers and the zeroes of $\zeta(2 s)$ in the strıp $0 \leqq \Re(s)<1 / 2$. 
From the functional equation of $\zeta$,

$$
\pi^{-\frac{1}{2} s} \Gamma\left(\frac{1}{2} s\right) \zeta(s)=\pi^{-\frac{1}{2}+\frac{1}{2} s} \Gamma\left(\frac{1}{2}-\frac{1}{2} s\right) \zeta(1-s)
$$

we have:

Proposition 2.1.5 (Functional Equation). The function $\mathcal{M}_{f}(s)$ satisfies the functional equation:

$$
\frac{\pi^{s-\frac{1}{2}} \mathcal{M}_{f}\left(\frac{1}{2}-s\right)}{\varphi_{f}\left(\frac{1}{2}-s\right) \Gamma\left(\frac{1}{2}-s\right) \zeta(-2 s)}=\frac{\pi^{-s} \mathcal{M}_{f}(s)}{\varphi_{f}(s) \Gamma(s) \zeta(2 s-1)}
$$

Suppose that $f \in C_{c}^{k}\left(\mathbb{R}^{\bullet}\right)$, for $k \geqq 1$. Then, integrating by parts we obtain:

$$
\varphi_{f}(s)=\frac{(-1)^{k}}{2 s(2 s+1) \ldots(2 s+k-1)} \int_{0}^{\infty} f^{(k)}(u) u^{2 s+k-1} d u .
$$

Therefore, if $f \in C_{c}^{k}\left(\mathbb{R}^{\bullet}\right)$, there exist positive constants $A$ and $B$ such that for all $\sigma \in \mathbb{R}$

$$
\left|\varphi_{f}(\sigma+i t)\right| \leqq A \frac{B^{2|\sigma|}}{(1+|t|)^{k}}
$$

The constants $A$ and $B$ depend only on $f$ and $k$. In fact, if $\operatorname{support}(f) \subset[a, b]$ and $0<a<b$, then

$$
\left|\varphi_{f}(\sigma+i t)\right| \leqq \frac{\left((b-a)\left\|f^{(k)}\right\|_{\infty} b^{k-1}\right) b^{|2 \sigma|}}{|2 \sigma+2 i t| \cdot|2 \sigma+1+2 i t| \ldots|2 \sigma+k-1+2 i t|} .
$$

Therefore, if $f \in C_{c}^{\infty}\left(\mathbb{R}^{\bullet}\right)$ it follows that $f$ belongs to the Paley-Wiener space, $P W(\mathbb{C})$ (see Lang $[\mathrm{L}]$, p. 74), i.e., there exists a constant $c>0$ such that for every natural number $N$ and $\sigma \in \mathbb{R}$ we have:

$$
\left|\varphi_{f}(\sigma+i t)\right| \ll \frac{c^{|2 \sigma|}}{(1+|t|)^{N}}, \quad \text { as } t \rightarrow \pm \infty .
$$

That is, given $N$ and $\sigma$ there exists a positive constant $K=K(f, N)$, depending only on $f$ and $N$ such that

$$
\left|\varphi_{f}(\sigma+i t)\right| \leqq K \frac{c^{|2 \sigma|}}{(1+|t|)^{N}}
$$

for all $t \in \mathbb{R}$ such that $|t| \geqq t_{0}$, where $t_{0}$ depends only on $f$ and $N$.

From (21) it follows that $\varphi_{f}$ is of rapid decay in any fixed vertical strip, i.e., $\varphi_{f}(\sigma+i t)$ tends very rapidly to zero uniformly in any strip $\sigma_{1} \leqq \sigma \leqq \sigma_{2}$, as $|t| \rightarrow \infty$. In particular, if $f \in C_{c}^{2}\left(\mathbb{R}^{\bullet}\right)$, we have that the function $g_{f}^{\sigma}: \mathbb{R}^{\bullet} \rightarrow \mathbb{C}$ defined by

$$
g_{f}^{\sigma}(t)=\varphi_{f}(\sigma+i t)
$$

has the property that $g_{f}^{\sigma} \in \mathcal{L}_{1}(\mathbb{R}, \mathbb{C})$, for all $\sigma \in \mathbb{R}$.

Now, let us recall the following facts about the order of growth of $\zeta(s)$ along vertical lines. Let $\mu(\sigma)$ be the lower bound of real numbers $\ell \geqq 0$ such that

$$
\zeta(\sigma+i t)=\mathcal{O}\left(|t|^{\ell}\right) \quad \text { as } \quad|t| \rightarrow \infty .
$$


Then $\mu$ has the following properties (Titchmarsh [T], p. 95):

$$
\begin{cases}\text { i) } & \mu \text { is continuous non-increasing and never negative. } \\ \text { ii) } & \mu \text { is convex downwards in the sense that the curve } y=\mu(\sigma) \\ & \text { has no points above the chord joining any two of its points. } \\ \text { iii) } & \mu(\sigma)=0 \text { if } \sigma \geqq 1 \text { and } \mu(\sigma)=\frac{1}{2}-\sigma \text { if } \sigma \leqq 0\end{cases}
$$

The Lindelöf hypothesis is equivalent to the statement that

$$
\begin{cases}\mu(\sigma)=\frac{1}{2}-\sigma & \text { if } \sigma<\frac{1}{2} \\ \mu(\sigma)=0 & \text { if } \sigma \geqq \frac{1}{2}\end{cases}
$$

which is equivalent to:

$$
\zeta\left(\frac{1}{2}+i t\right)=\mathcal{O}\left(t^{\epsilon}\right) \text { for all } \epsilon>0
$$

Now suppose the Riemann hypothesis is true; then $\log \zeta(s)$ is a holomorphic function in the half-plane $\Re(s)>\frac{1}{2}$ (except at $s=1$ ) and we have the following estimates due to Littlewood:

$$
\begin{aligned}
& \text { For } \epsilon>0 \text { and } \sigma \geqq \frac{1}{2}: \\
& -\epsilon \log t<\log |\zeta(s)|<\epsilon \log t ; \quad s=\sigma+i t, \quad t \geqq t_{0}(\epsilon),
\end{aligned}
$$

that is:

$$
\left\{\begin{array}{l}
\zeta(s)=\mathcal{O}\left(t^{\epsilon}\right) \\
\frac{1}{\zeta(s)}=\mathcal{O}\left(t^{\epsilon}\right)
\end{array} \quad \text { for every } \epsilon>0, s=\sigma+i t, \sigma>\frac{1}{2} \text { as }|t| \rightarrow \infty\right.
$$

The estimates (27), valid under the Riemann hypothesis, can be found in Titchmarsh [T], Chapter XIV, p. 337, formulæ (14.2.5) and (14.2.6). Furthermore, suppose that $\alpha>\frac{1}{2}$ is such that $\zeta$ has no zeroes in the half-plane $\Re(s)>\alpha$; then Littlewood has the following estimates:

$$
\left\{\begin{array}{l}
\zeta(s)=\mathcal{O}\left(t^{\epsilon}\right) \\
\frac{1}{\zeta(s)}=\mathcal{O}\left(t^{\epsilon}\right)
\end{array} \text { for every } \epsilon>0, s=\sigma+i t, \sigma \geqq \alpha .\right.
$$

Also if for each $\sigma>\frac{1}{2}$ (and $s=\sigma+i t$ as before) we define $\nu(\sigma)$ as the lower bound of numbers $a$ such that

$$
\log \zeta(s)=\mathcal{O}\left(\log ^{a} t\right)
$$

then for $\beta<\sigma<1, \beta=\sup \{\Re(\rho) \mid \zeta(\rho)=0\}$, we have

$$
1-\sigma \leqq \nu(\sigma) \leqq 2(1-\sigma) .
$$

Also $\log \zeta(s)$ (for $\Re(s)>\beta$ ) has the same $\nu$ function as $\frac{\zeta^{\prime}(s)}{\zeta(s)}$, i.e., if we define $\nu^{\prime}(\sigma)$ as the lower bound of numbers such $a$ that

$$
\frac{\zeta^{\prime}(s)}{\zeta(s)}=\mathcal{O}\left(\log ^{a} t\right)
$$

then

$$
1-\sigma \leqq \nu^{\prime}(\sigma) \leqq 2(1-\sigma)
$$


Also, (see Titchmarsh, Chapter XIV, Theorem 14.5) we have:

$$
\frac{\zeta^{\prime}(s)}{\zeta(s)}=\mathcal{O}\left((\log t)^{2-2 \sigma}\right)
$$

uniformly for $\beta<\sigma_{0} \leqq \sigma \leqq \sigma_{1}<1, \sigma \neq \frac{1}{2}$.

\section{Proof of Theorems}

In all that follows we will assume that $f \in C_{c}^{\infty}\left(\mathbb{R}^{\bullet}\right)$ but everything will still hold if we only assume that $f \in C_{c}^{r}\left(\mathbb{R}^{\bullet}\right), r \geqq 2$.

3.1. Proof of Theorem A. By the Mellin inversion formula we have:

$$
m_{y}(f)=\frac{1}{2 \pi i} \int_{a-\imath \infty}^{a+\imath \infty} \mathcal{M}_{f}(s) y^{1-s} d s
$$

for an appropriate $a \in \mathbb{R}$. In our case we can take $a=\frac{1}{2}$ because the function $\Theta_{\frac{1}{2}}(t)=$ $\mathcal{M}_{f}\left(\frac{1}{2}+i t\right)$ satisfies $\Theta_{\frac{1}{2}} \in \mathcal{L}_{1}(\mathbb{R}, \mathbb{C})$; this is so because the function $\varphi_{f}\left(\frac{1}{2}+i t\right)$ is in the Paley-Wiener space and by $(24)$ the function $Z_{f}(t)=\frac{\zeta(2 i t)}{\zeta(1+2 i t)}$ is $\mathcal{O}\left(|t|^{\frac{1}{2}+\epsilon}\right)$ for all $\epsilon>0$. Hence $\left.\varphi_{f}\right|_{\Re(s)=\frac{1}{2}} \cdot Z_{f} \in \mathcal{L}_{1}(\mathbb{R}, \mathbb{C})$.

The integral of $\mathcal{M}_{f}(s) y^{1-s}$ over the boundary of of the vertical strip $\frac{1}{2} \leqq \sigma \leqq 2$ exists and it is equal at the same time to $\mathcal{R} e s_{s=1}\left(\mathcal{M}_{f}(s)\right)$ and equal to

We have:

$$
m_{y}(f)-\frac{1}{2 \pi} \int_{-\infty}^{\infty} \mathcal{M}_{f}\left(\frac{1}{2}+i t\right) y^{\frac{1}{2}} y^{-i t} d t
$$

$$
\left|\int_{-\infty}^{\infty} \mathcal{M}_{f}\left(\frac{1}{2}+i t\right) y^{\frac{1}{2}} y^{-i t} d t\right|=y^{\frac{1}{2}}\left|\int_{-\infty}^{\infty} \mathcal{M}_{f}\left(\frac{1}{2}+i t\right) y^{-i t} d t\right|=o\left(y^{\frac{1}{2}}\right)
$$

because, by the Riemann-Lebesgue Theorem:

Thus

$$
\lim _{y \rightarrow 0}\left|\int_{-\infty}^{\infty} \mathcal{M}_{f}\left(\frac{1}{2}+i t\right) y^{-i t} d s\right|=0
$$

$$
m_{y}(f)=\frac{1}{\zeta(2)} \int_{0}^{\infty} u f(u) d u+o\left(y^{\frac{1}{2}}\right)
$$

This proves theorem A.

3.2. Proof of Theorem B. Suppose the Riemann hypothesis is true. Then we set in formula (29) $a=\frac{1}{4}+\epsilon$, for any fixed $\epsilon>0$. Then the function $\Theta_{\frac{1}{4}+\epsilon}(t)=\mathcal{M}_{f}\left(\frac{1}{4}+i t\right)$ has the property that $\Theta_{\frac{1}{4}+\epsilon} \in \mathcal{L}_{1}(\mathbb{R}, \mathbb{C})$.

Therefore, the integral of $\mathcal{M}_{f}(s) y^{1-s}$ exists over the boundary of the band $\frac{1}{4}+\epsilon \leqq$ $\sigma \leqq 2$. Therefore:

$$
m_{y}(f)=\mathcal{R} e s_{s=1}\left(\mathcal{M}_{f}(s)\right)+\frac{1}{2 \pi} \int_{-\infty}^{\infty} \mathcal{M}_{f}\left(\frac{1}{4}+\epsilon+i t\right) y^{-i t} y^{\frac{3}{4}-\epsilon} d t
$$

Again, by the Riemann-Lebesgue Theorem:

$$
m_{y}(f)=\frac{1}{\zeta(2)} \int_{0}^{\infty} u f(u) d u+o\left(y^{\frac{3}{4}-\epsilon}\right)
$$


If, on the other hand, $m_{y}(f)=\frac{1}{\zeta(2)} \int_{0}^{\infty} u f(u) d u+o\left(y^{\frac{3}{4}-\epsilon}\right)$ for all $\epsilon>0$ and all functions $f \in C_{c}^{\infty}\left(\mathbb{R}^{\bullet}\right)$, then $\mathcal{M}_{f}(s)$ is holomorphic (except for a pole at $s=1$ ) in the half-plane $\Re(s)>\frac{1}{4}+\epsilon$, for all $\epsilon>0$. Since, under the hypotheses, $\mathcal{M}_{f}(s)=\frac{2 \zeta(2 s-1)}{\zeta(2 s)} \varphi_{f}(s)$ is holomorphic in that half-plane and we can choose $f$ so that $\varphi_{f}(s)$ does not vanish at any given zero of $\zeta$, it follows that $\zeta(2 s-1) / \zeta(2 s)$ is holomorphic in the half-plane $\Re(s)>\frac{1}{4}$ and hence the Riemann hypothesis would be true. The reason that $\mathcal{M}_{f}(s)$ is holomorphic in the half-plane, under the hypothesis that $m_{y}(f)=m_{0}(f)+K(y)$, where $K(y)=o\left(y^{\frac{3}{4}-\epsilon}\right)$, is the following:

$$
\mathcal{M}_{f}(s)=\frac{m_{0}(f)}{s-1}+\int_{0}^{\infty} K(y) y^{s-2} d y
$$

The integral in the right-hand side of (32) converges absolutely and uniformly in the half-plane $\Re(s)>\frac{1}{4}+\epsilon$, so it defines a holomorphic function in that half-plane.

Suppose that $\beta=\sup \{\Re(\rho) \mid \zeta(\rho)=0\}$. Then the function $\Theta_{\epsilon}(t)=\mathcal{M}_{f}\left(\frac{\beta}{2}+\epsilon+i t\right)$ belongs to $\mathcal{L}_{1}(\mathbb{R}, \mathbb{C})$ for all $\epsilon>0$.

This fact follows from (24), and the fact that $\varphi_{f}(s)$ is of rapid decay on vertical lines. As we know, $\frac{\beta}{2} \in\left[\frac{1}{4}, \frac{1}{2}\right]$ since Riemann's zeta-function has an infinite number of zeroes on the line $\Re(s)=\frac{1}{2}$ (Littlewood, Titchmarsh, Landau, Selberg) and no zeroes on the closed half-plane $\Re(s) \geqq 1$ (by the prime number theorem). Then, by the Mellin inversion formula we have:

$$
m_{y}(f)=m_{0}(f)+o\left(y^{1-\frac{\beta}{2}-\epsilon}\right) \quad \text { for all } \epsilon>0 .
$$

If, on the other hand, (33) holds with $\alpha=1-\frac{\beta}{2}-\epsilon, \frac{\beta}{2} \in\left[\frac{1}{4}, \frac{1}{2}\right)$ then, proceeding as in the proof of formula (32) we obtain that $\zeta$ has no zeroes in the half-plane $\Re(s)>2(1-\alpha)$.

Therefore, we have proven everything stated in Theorem B except for the fact that the exponent $\frac{1}{2}$ is optimal for characteristic functions and the assertion regarding the function $F$. To finish the proof we need the following: For $x>0$, let $\Phi(x)=\sum_{n \leqq x} \varphi(n)$ and set $\Phi(x)=0$ for $0<x<1$. Then by Mertens theorem $\Phi(x)=\frac{3}{\pi^{2}} x^{2}+(x \log x) b(x)$ for a bounded function $b(x):-c<b(x)<c$ for all $x>0$ and some constant $c>0$.

LEMMA 3.2.1. For all $\alpha>1$

$$
\varlimsup_{x \rightarrow \infty} x^{\alpha}\left|\frac{\Phi(x)}{x^{2}}-\frac{3}{\pi^{2}}\right|=\infty
$$

Proof. Suppose the contrary. Then there exist $\alpha>1, c>0$ and a function $b_{\alpha}(x)$ defined on the positive reals and such that $-c<b_{\alpha}(x)<c$ for all $0<x<\infty$ such that

$$
\frac{\Phi(x)}{x^{2}}-\frac{3}{\pi^{2}}=x^{-\alpha} b_{\alpha}(x) \text {. }
$$

Let $H(x)=\Phi(x) / x^{2}$. Then

$$
H(x+1)=\Phi(x) \frac{x^{2}}{(x+1)^{2}}+\frac{\varphi([[x+1]])}{(x+1)^{2}},
$$

where $[[\cdot]]$ denotes integral part.

By (34) and (35) and letting $x$ run over the integers such that $x+1$ is a prime, we obtain:

$L(x)=x^{\alpha}\left[\frac{3}{\pi^{2}}\left[\frac{2 x-1}{(x+1)^{2}}\right]-\frac{x}{(x+1)^{2}}\right]=b_{\alpha}(x)\left[\frac{x^{2}}{(x+1)^{2}}\right]-b_{\alpha}(x+1)\left[\frac{x}{x+1}\right]^{\alpha}=R(x)$ 
But this is an absurdity since $L(x)$ is unbounded when $\alpha>1$ whereas the right-hand side remains bounded. This proves the statement in Theorem B for the characteristic function of the interval $(0,1]$. The proof for an arbitrary closed interval is similar. Now let $F$ be the function given in Theorem $\mathrm{B}$ and suppose:

$$
\varlimsup_{y \rightarrow 0} y^{-\alpha}\left|m_{y}(F)-m_{0}(F)\right|=\infty, \quad \text { for all } \alpha>\frac{1}{2}
$$

First we note that

$$
m_{0}(F)=\frac{1}{\zeta(2)} \int_{0}^{\infty} u F(u) d u=\frac{1}{\pi^{2}}
$$

The Mellin transform $M_{F}(s)$ is:

$$
\mathcal{M}_{F}(s)=\frac{\zeta(2 s-1)}{s(2 s+1) \zeta(2 s)}
$$

Hence its only poles in the half-plane $\Re(s)>0$ are located at the zeroes of $\zeta(2 s)$, since $s(2 s+1)$ does not vanish in that half-plane. $\mathcal{M}_{F}(s)$ is not of rapid decay namely not of Paley-Wiener type, however it decays fast enough so as to be able to shift the vertical line of integration-in Mellin's inversion formula - to the vertical line $\Re(s)=\frac{\beta}{2}+\epsilon$ where $\beta$, as before, is the supremum of the real parts of the zeroes of Riemann's $\zeta$-function. Now suppose $\beta<1$. We want to arrive to a contradiction.

First we note that for any $\epsilon>0$ the inequalities (24) through $\left(28^{\prime \prime}\right)$ imply that the function

$$
h(t)=\frac{\zeta(\beta-1+2 \epsilon+2 i t)}{(\beta / 2+\epsilon+i t)(1+\beta+2 \epsilon+2 i t) \zeta(\beta+2 \epsilon+2 i t)}
$$

has the property that

$$
\lim _{|t| \rightarrow \infty} h(t)=0
$$

and

$$
\lim _{|t| \rightarrow \infty} h^{\prime}(t)=0
$$

for all $\epsilon>0$. In fact we have if $s=\sigma+i t$ and $-1 \leqq \sigma \leqq 2$ :

$$
\left|\zeta^{\prime}(s)\right| \leqq K_{1}|\log t||t|^{\mu(\sigma)}
$$

and

$$
|\zeta(s)| \leqq K_{2}|t|^{\mu(\sigma)}
$$

for some constants $K_{1}, K_{2}>0$ and $|t|$ sufficiently large. Hence under the hypotheses:

$$
\begin{aligned}
|h(t)| & \leqq K_{1}|t|^{-\delta} \\
\left|h^{\prime}(t)\right| & \leqq K_{2}|t|^{-\delta}
\end{aligned}
$$

for some $\delta>1$. On the other hand, the improper integral

exists for all $y>0$. Namely

$$
\frac{1}{2 \pi} \int_{-\infty}^{\infty} h(t) y^{1-\frac{\beta}{2}-\epsilon-i t} d t
$$

$$
\lim _{T_{1}, T_{2} \rightarrow \infty} \frac{1}{2 \pi} \int_{-T_{1}}^{T_{2}} h(t) y^{1-\frac{\beta}{2}-\epsilon-\imath t} d t
$$


exists for all $y>0$, This follows from Cauchy's Residue Theorem by integrating the function $H_{y}(s)=\frac{1}{2 \pi \imath} \mathcal{M}_{F}(s) y^{1-s}$ for each fixed $y>0$ along the rectangle $Q\left(T_{1}, T_{2}\right)$ with vertices:

$$
\begin{aligned}
& A\left(T_{1}\right)=\frac{\beta}{2}+\epsilon-i T_{1} \\
& B\left(T_{2}\right)=\frac{\beta}{2}+\epsilon+i T_{2} \\
& C\left(T_{2}\right)=2+i T_{2} \\
& D\left(T_{1}\right)=2-i T_{1} .
\end{aligned}
$$

The integrals along the segments $\left[B\left(T_{2}\right), C\left(T_{2}\right)\right]$ and $\left[D\left(T_{1}\right), A\left(T_{1}\right)\right]$ tend uniformly to zero as $T_{1}$ and $T_{2}$ tend to infinity, hence

$$
\lim _{T_{1}, T_{2} \rightarrow \infty} \frac{1}{2 \pi} \int_{-T_{1}}^{T_{2}} h(t) y^{1-\frac{\beta}{2}-\epsilon-\imath t} d t=-\frac{1}{\pi^{2}}+m_{y}(f) .
$$

Hence:

$$
m_{y}(F)=\frac{1}{\pi^{2}}+\frac{1}{2 \pi} y^{1-\frac{\beta}{2}-\epsilon} \int_{-\infty}^{\infty} h(t) y^{-i t} d t
$$

Now consider the integral

$$
G(y)=\int_{-\infty}^{\infty} h(t) e^{-(\log y) i t} d t .
$$

By (42), $G(y)$ is continuous. Also, $G(y)=\widehat{h}(\log y)$ where $\hat{h}$ is the Fourier transform of $h$. Since $h \in \mathcal{L}_{1}(\mathbb{R}, \mathbb{C}) h$ has a well defined Fourier transform and all of the above is valid. In fact since $h$ and $h^{\prime}$ vanish at infinity, we have for $y \neq 1$ :

$$
G(y)=i[\log y]^{-1} \lim _{a \rightarrow \infty} \int_{-a}^{a} e^{-(\log y) i t} d h
$$

Integration by parts is valid, since both $h$ and $h^{\prime}$ vanish at infinity. Hence RiemannLebesgue is valid and we obtain:

$$
\lim _{y \rightarrow 0} G(y)=0 .
$$

Therefore, under the hypothesis $\beta<1$ we obtain:

and therefore:

$$
m_{y}(F)=\frac{1}{\pi^{2}}+\frac{1}{2 \pi} y^{1-\frac{\beta}{2}-\epsilon} G(y) \text { for all } \epsilon>0
$$

$$
\lim _{y \rightarrow 0} y^{-1+\frac{\beta}{2}+\epsilon}\left|m_{y}(F)-\frac{1}{\pi^{2}}\right|=0 .
$$

But this contradicts the hypothesis since $1-\frac{\beta}{2}-\epsilon>\frac{1}{2}$ if $\epsilon$ is small enough.

Remark. We have shown that:

$$
m_{y}(F)=\frac{1}{\pi^{2}}+o\left(y^{1 / 2}\right)
$$

Now let $y=N^{-2}$ where $\mathrm{N}$ is a positive integer. Then,

$$
m_{y}(F)=N^{-3} \sum_{n=1}^{N-1} \Phi(n)
$$


From the last two equations we obtain:

$$
\lim _{N \rightarrow \infty} N^{-3} \sum_{n=1}^{N-1} \Phi(n)=\frac{1}{\pi^{2}}
$$

and,

$$
\lim _{N \rightarrow \infty}\left[N^{-2} \sum_{n=1}^{N-1} \Phi(n)-\frac{N}{\pi^{2}}\right]=0
$$

By Mertens Theorem, one has:

$$
\sum_{n=1}^{N-1} \Phi(n)=\frac{3}{\pi^{2}} \sum_{n=1}^{N-1} n^{2}+\sum_{n=1}^{N-1} n b(n) \log (n)
$$

For some bounded function $b(n)$. Hence, recalling that $\sum_{n=1}^{N-1} n^{2}=\frac{2 N^{3}-3 N^{2}+N}{6}$, we obtain:

Corollary.

$$
\lim _{N \rightarrow \infty} N^{-2} \sum_{n=1}^{N-1} n b(n) \log (n)=\frac{3}{2 \pi^{2}}
$$

Acknowledgements. I would like to thank E. Ghys, S. Lopez de Medrano, C. McMullen, B. Randol, D. Sullivan and D. Zagier. I would also like to thank the organizers of the International Workshop on Topology and Geometry held in Hanoi, Vietnam.

\section{REFERENCES}

[A] T. Apostol, Introduction to analytic number theory, GTM Springer-Verlag, Berlin, Heidelberg, New York, 1985.

[B] S. Bochner, Lectures on Fourier Integrals, Annals of Mathematical Studies 42, Princeton Univ. Press, New Jersey, 1959.

[Fr] J. Franel, Les Suites de Farey et le problème des nombres premiers, Göttinger Nachrichten (1924), 198-201.

[G] E. Ghys, Actions localment libres du groupe affine, Inv. Math.82 (1985), 479-526.

[HW] G.H. Hardy and E.M. Wright, An introduction to the theory of numbers, Oxford Univ. Press, London, 1975.

[L] S. Lang, $S L_{2}(\mathbb{R})$, Addison-Wesley, Reading, Mass., 1975.

[La] E. Landau, Bemerkung zu der vorstehenden Arbeit von Herrn Franel, Göttinger Nachrichten (1924), 202-206.

[S] P. Sarnak, Asymptotic behaviour of periodic orbits of the horocycle flow and Eisenstein series, Comm. in Pure and App. Math. 34 (1980), 719-739.

[T] E.C. Titchmarsh, The theory of Riemann zeta-function, Oxford Univ. Press, London, 1988.

[V] A. Verjovsky, Arithmetic, Geometry and dynamics in the modular orbifold, Dynamical Systems, (Santiago de Chile 1990) (Pitman Series 285), R. Bamon, R. Labarca, J. Lewowicz, J. Palis, Longman, Essex, UK, 1992, pp. 263-298.

[Z] D. Zagier, Eisenstein Series and the Riemann zeta function, Automorphic forms, Representation theory and Arithmetic, Tata Institute of Fundamental Research, Bombay, 1979, Springer-Verlag, Berlin, Heidelberg, New York, 1981, pp. 275-301. 
Departement de Mathematiques

UNIVERSITE DE SCIENCES ET

TECHNOLOGIES DE LILLE

VILLENEUVE D'ASCQ 59655

France

e-mail: Alberto@gat.citilille.fr 